\title{
Investigation of ground water table in the South-East (Chittagong) part of Bangladesh
}

\author{
Md. Abdul Hamid Mirdad, Swapan Kumar Palit \\ Department of Civil Engineering, Chittagong University of Engineering \& Technology (CUET), Chittagong, Bangladesh
}

\section{Email address:}

adnan_cuet@yahoo.com (Md. A. H. Mirdad), skpbd@cuet.ac.bd (S. K. Palit)

\section{To cite this article:}

Md. Abdul Hamid Mirdad, Swapan Kumar Palit. Investigation of Ground Water Table in the South-East (Chittagong) Part of Bangladesh. American Journal of Civil Engineering. Vol. 2, No. 2, 2014, pp. 53-59. doi: 10.11648/j.ajce.20140202.17

\begin{abstract}
Ground water forms the major portion of earth's fresh water source and it is almost safe to drink. Depletion of ground water table due to continuous pumping causing scarcity of water in the city area of Bangladesh. So information about ground water table is required for future recommendation of ground water supply to general people. For the investigation purpose, depth of water table has been determined in three seasons with respect to mean sea level. Among 41 wards of Chittagong City, 18 wards have been selected for this purpose where depth of water table is measured from the shallow tube well. The present investigation includes field investigation for locating of tube well in Chittagong City Corporation area with the aim of measuring water level from ground surface. Seasonal variation of the water level has also been determined with respect to man sea level. Water collected from the well has been evaluated in the laboratory for determining quality of water. From the investigation, it has been established that, water table with respect to mean sea level is different at different wards. Seasonal variation has been observed due to lowering of water table and it has been found that water table is minimum at drought season.
\end{abstract}

Keywords: Ground Water, Depletion of Ground Water, Sea Level, Seasonal Variation, Quality Water, Drought

\section{Introduction}

Ground water forms the major portion of earth's fresh water supply. About $97 \%$ of the earth's fresh water supply is stored in the underground formation with the increase in population, the demand for water system is essential to meet the increasing demand for water is also increasing throughout the world. Effective management of ground water system in essential to meet the increasing demand for water. Ground water can be used as a reliable source of water supply irrespective of the climate. In the monsoon areas of South-East Asia, ground water way becomes an important source of supply especially for irrigation purposes in the dry months. $94 \%$ of all the water-works use ground water and they supply $77 \%$ of the total water consumed. Ground water is a vital source of water supply, especially in area where dry summers or extended droughts cause stream flow to stop water from the surface streams is the main source of recharge for the ground water (Aziz, 1975).

\subsection{Ground Water}

Ground water is water located beneath the ground surface in soil pore spaces and in the fractures of rock formations. A unit of rock or an unconsolidated deposit is called an aquifer when it can yield a usable quantity of water. The depth at which soil pore spaces or fractures and voids in rock become completely saturated with water is called the water table. Groundwater is recharged from and eventually flows to the surface naturally, natural discharge often occurs at springs and seeps, a can form oases or wetlands. Ground water is also often withdrawn for agricultural, municipal $\&$ industrial use by constructing \& operating extraction wells. The study of the distribution and movement of ground water is hydrogeology, also called ground water hydrology.

\subsection{Ground Water Situation in Bangladesh}

Ground water is a vital source of water supply for Bangladesh. Bangladesh is almost entirely underlain by water-bearing formations at depths varying from zero to 20 $\mathrm{m}$ below ground surface. The soil is mostly stratified and formed by alluvial deposits of sand and silt, having 
occasional limes of clay. The main constituent of the aquifer materials is the medium grained sand deposited at the lower reach by the mighty rivers the Ganges, the Brahmaputra and the Meghna with their tributaries. (Aziz, 1975). Ground water in Bangladesh, except in some places, is available at a shallow depth. Ground water levels are at or near ground level during the period August-October and lowest in April-May. Ground water rives as a result of recharge during April-May and usually reaches its highest in late July in each year. Between July and October ground water level are constant and maintain a balance between surface water levels and the fully recharged aquifers. There are several areas of Bangladesh where ground water withdrawals are causing a large deceive in ground water level during dry seasons. The ground water withdrawal and recharge characteristics suggest that the actual recharge can be increased approaching the potential limits by creating addition storage through increased abstraction during the dry season. According to MPO (1991) estimates, out of $42543 \mathrm{Mm}^{3}$ total useable recharge, $40 \%$ is available through shallow tube wells. A study shows that 349 Upazila can adequately allow ground water development by hand pump tube wells of which 197 Upazila having ground water level within $4.5 \mathrm{~m}$ of the surface in dry season allows groundwater abstraction by suction mode No. 6 pump. In 60 Upazila, the groundwater level lies within $4.5 \mathrm{~m}$ to 6.5 $\mathrm{m}$, the marginal range for forced mode Tara Pump. In 92 Upazilas the water level falls below the limit of suction mode pumps in the later part of dry season.

\subsection{The Study Area}

Chittagong city is the second largest city of Bangladesh, considered the heart of all commercial and business activity. At present, the city's land area occupies around $157 \mathrm{~km}^{2}$ inhabited by about 4 million people (2001 census). Chittagong Water Supply and Sewerage Authority (CWASA) which is the authority for water supply and sewerage only supply water to one-third of city dwellers. Rest of people depends on the shallow tube well and deep tube well. Location of ground water table in Chittagong City Corporation area has been shown in study area map of Figure 1. For the investigation purpose specific problem has been identified and through which important information can be found.

\subsection{Ground Water Table in Chittagong City According to CWASA}

Ground water table in Chittagong City is at present in a position from where it is tough to pump ground water by shallow tube well. Deep tube well is required almost in every position to find the fresh water from ground. According to Chittagong WASA they have 89 deep tube well permanently in all city area. Location \& depth of all the deep tube well has not been found as the authority of CWASA provides location and depth of some deep tube well. Location and depth of some deep tube well of CWASA are shown in Table 1.
Table 1. Location \& depth of deep tube well of CWASA.

\begin{tabular}{lll}
\hline Location & Depth (m) & Depth (ft.) \\
\hline 1. Head of Kaptai Road (13) & $165.7 \mathrm{~m}$ & $543.5 \mathrm{ft}$ \\
2. Asian Housing Society (22) & $175 \mathrm{~m}$ & $574 \mathrm{ft}$ \\
3. Moulabi Pukur Par (08) & $141.7 \mathrm{~m}$ & $464.1 \mathrm{ft}$ \\
4. Agrabad Al Nahian, CDA & $296.62 \mathrm{~m}$ & $973 \mathrm{ft}$ \\
5. Halishahar Housing \& Settlement & $298.12 \mathrm{~m}$ & $977.8 \mathrm{ft}$ \\
School, L Block & $196.62 \mathrm{~m}$ & $645 \mathrm{ft}$ \\
6. Bishaw Colony, D Block & $134.83 \mathrm{~m}$ & $442.2 \mathrm{ft}$ \\
7. Khawaja Road (3) & $208.90 \mathrm{~m}$ & $685.2 \mathrm{ft}$ \\
8. Dewanhat CSD Gudam & $111.81 \mathrm{~m}$ & $366.7 \mathrm{ft}$ \\
9. Salimpur Housing Society, Foujderhat & $190.32 \mathrm{~m}$ & $624.2 \mathrm{ft}$ \\
\hline
\end{tabular}

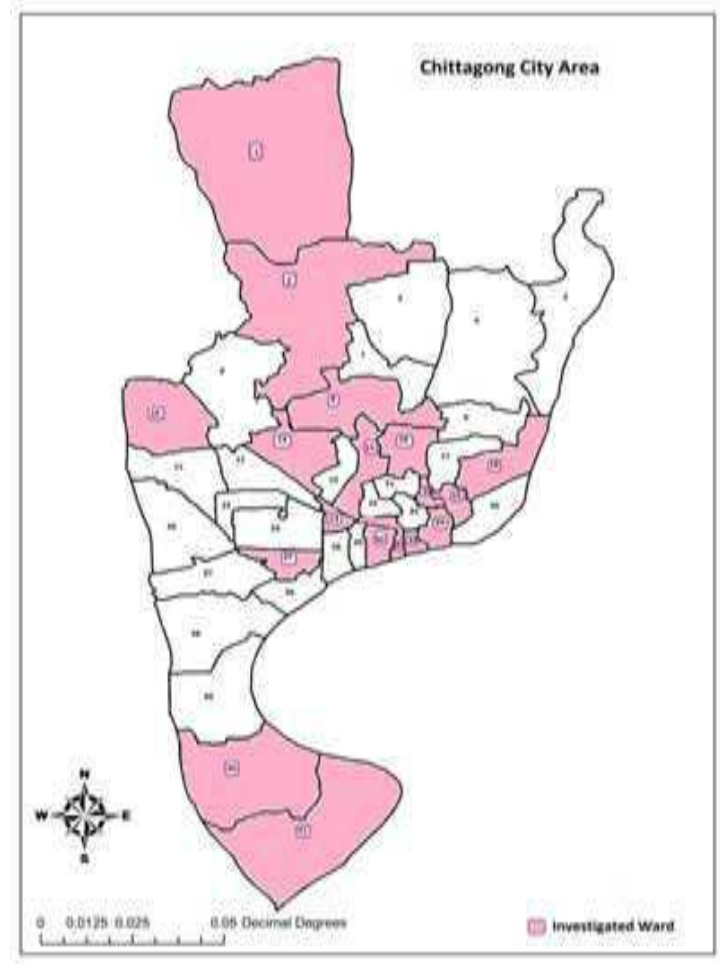

Figure 1. Study area map of Chittagong City Corporation.

\section{Methodology}

Investigation of ground water table in Chittagong City is overall a tough ask. Although the water level in Chittagong City is in a critical state, an investigation has been done with great consciousness.

\subsection{Field Investigation for Shallow Well}

At the beginning of the investigation first task is to find out the shallow tube well in the 41 ward's City Corporation School. For this school list has been taken from City Corporation. Following the list to school, location of shallow tube well is found. Maps of Chittagong district has been shown in Figure 2. 


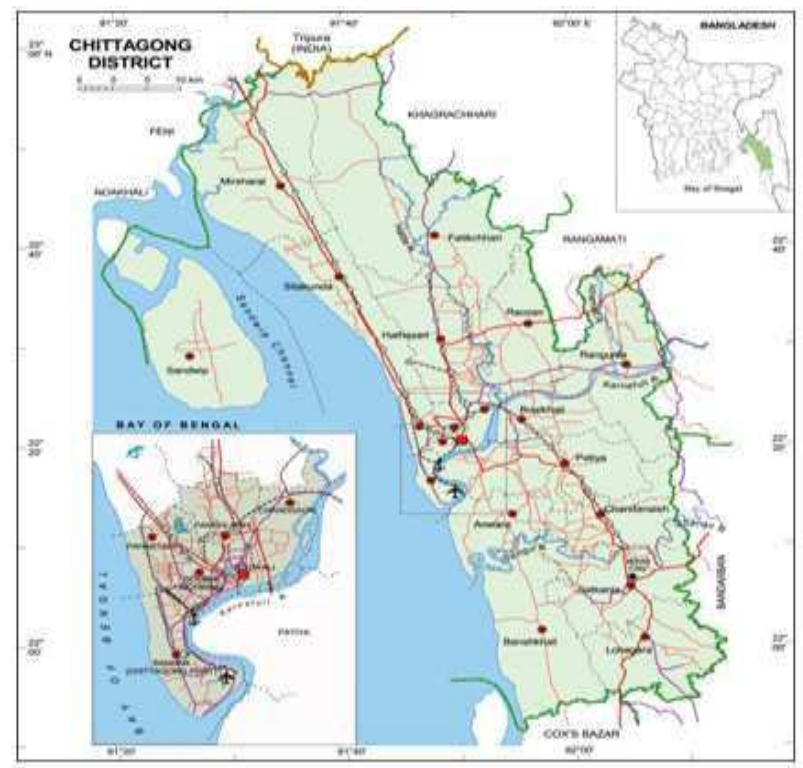

Figure 2. Maps of Chittagong district.

\subsection{Reduce Level in Well Location}

It is too tough to find out reduce level of well location with respect to mean sea level. But by using Global Positioning System (GPS), Reduce level of ground surface in well location can be easily found. GPS is a space based global navigation satellite system that provides reliable location and time information in all weather $\&$ all times anywhere on or near the earth when $\&$ where there is an unobstructed line of sight to four or more GPS satellites. On the well location, switch on the GPS. Then wait for five minutes to set its location properly. Then it will give value of longitude latitude \& elevation of that specific location with respect to mean sea level. Determination of reduce level in well location with GPS device has been shown in Figure 3. GPS device gives almost accurate elevation in the location. GPS device works accurately in the open area.

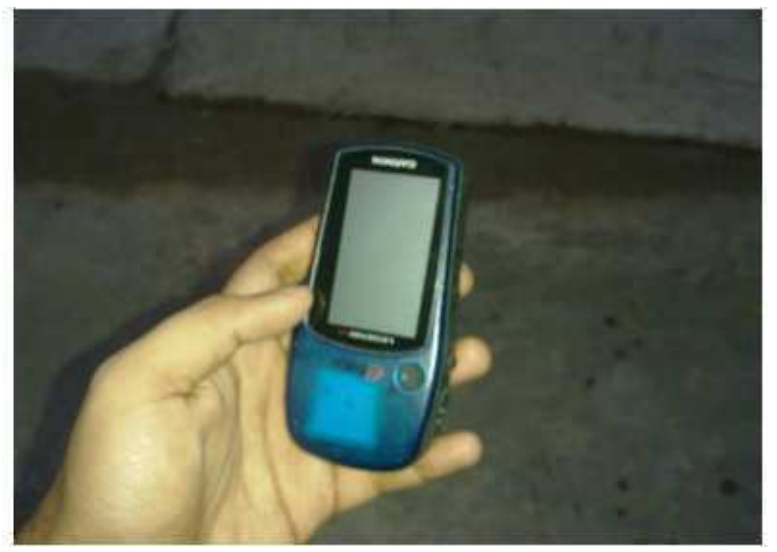

Figure 3. Determination of RL in well location by GPS device.

\subsection{Depth of Water Table in Different Season}

Depth of water table has been measured from the ground level at well location by opening the head of well. After opening the head of the well, wait for 10-15 minutes to drop the water in the well pipe. Then using a thick wire with a small steal at its bottom, entering into the well pipe until the wire is in a state of less weight. Then by using tape, find the depth from the weir. In this process, the depth is measured three times. Once at the beginning of winter season (09.11.2010) when the water table is fully saturated, once at the drought season (07.04.2011) when the water table is in lowest position as suction of water is more and once at the beginning of rainy season (31.05.2011) after some rainfall when water table is recharged and comes up.

\subsection{Water Quality Parameter Test of Sample}

Different water quality parameter such as $\mathrm{pH}$, Iron, Alkalinity, Arsenic, $\mathrm{CO}_{2}$ and Salinity has been determined in the laboratory from the sample water collected from well location. After determination compare the result with Bangladesh standard and WHO standard and suggest weather the water is drinkable or not. Water quality parameter test done in the Environmental laboratory has been shown in Figure 4. Arsenic has been tested by Wagtech Arsenic kit box. In each test it takes 25 minutes to find the result by comparing the filter paper with the color chart. Test done by Wagtech Arsenic kit box which gives almost accurate values in the field. Wagtech Arsenic kit box with sample water collected from well location has been shown in Figure 5.

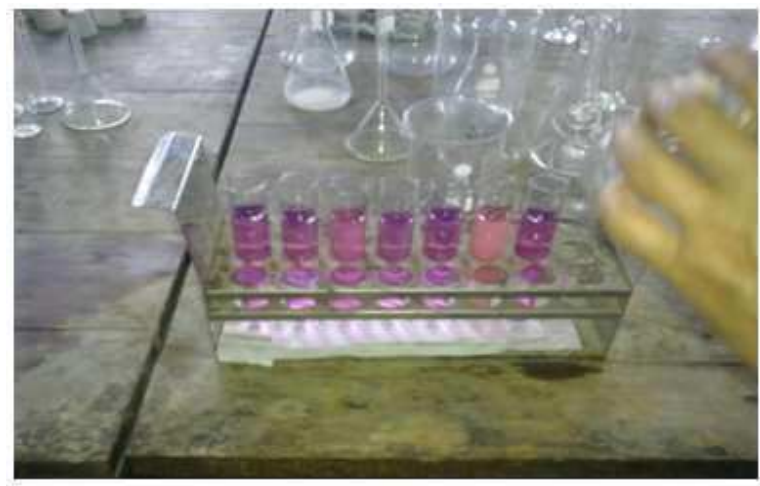

Figure 4. Water quality parameter test in laboratory.

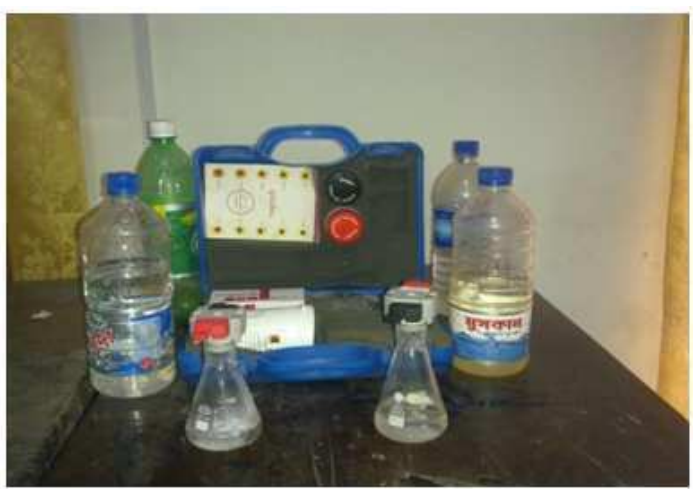

Figure 5. Wagtech Arsenic kit box with sample water. 


\section{Ground Water Table Investigation}

\subsection{Field Investigation for Location of Shallow Tube Well in Chittagong City}

According to the list of school from Chittagong City
Corporation, field investigation for the location of shallow tube well has been done. Location of shallow tube well and deep tube well in 41 ward's City Corporation school are given in Table 2. School well has been selected for the investigation because the well in school is permanent rather than the outside open well which may not present in future.

Table 2. Location of shallow and deep tube well in 41 wards City Corporation School.

\begin{tabular}{|c|c|c|c|}
\hline Ward No. & Name of the ward & Well location & Remarks \\
\hline \multirow[t]{2}{*}{01} & South Pahartali & Fatehabad Bohumuki City Corporation Girl's High School & Shallow tube well \\
\hline & & Kulgoan City Corporation High School & Shallow tube well \\
\hline \multirow[t]{3}{*}{02} & Jalabad & Imaratonnesa City Corporation Girl's High School & Shallow tube well \\
\hline & & East Nasirabad A. Jalil Govt. Primary School & Deep tube well. \\
\hline & & Panchlaish City Corporation Girl's High School & Deep tube well. \\
\hline \multirow[t]{2}{*}{03} & Panchlaish & & \\
\hline & & Sohid Dr. Mokbul Ahmed Govt. Primary School. & Deep tube well. \\
\hline 04 & Chandgaon & Mohammadpur Govt. Primary School & Deep tube well. \\
\hline \multirow[t]{2}{*}{05} & Mohara & & \\
\hline & & Kapashgola City Corporation Girl's High School & Deep tube well. \\
\hline \multirow[t]{3}{*}{06} & East Sholashahar & Kapashgola Primary School & Deep tube well. \\
\hline & & Eklasur Rahman Govt. Primary School & Deep tube well. \\
\hline & & Rahmania High School & Deep tube well. \\
\hline \multirow[t]{3}{*}{07} & West Sholashahar & Hamzarbag UCEF School & Deep tube well. \\
\hline & & Mohammadpur Govt. Primary School & Deep tube well. \\
\hline & & Hossain Ahmed Chowdhury City Corporation School & Shallow tube well \\
\hline \multirow[t]{2}{*}{08} & Sulakbahar & & \\
\hline & & Ahmed Miah Primary School & Deep tube well. \\
\hline 09 & North Pahartali & & \\
\hline 10 & North Kattali & Kattali City Corporation Girl's High School & Shallow tube well \\
\hline 11 & South Kattali & & \\
\hline 12 & Saraipara & Saraipara City Corporation School \& College. & Deep tube well \\
\hline 13 & Pahartali & New Jawtala Primary School & Shallow tube well \\
\hline \multirow[t]{2}{*}{14} & Lalkhan Bazar & Shahid Nagar City Corporation Girl's High School & Deep tube well \\
\hline & & Bagmoniram Abdur Rashid City Corporation Boy's High School & Shallow tube well \\
\hline \multirow[t]{2}{*}{15} & Bagmoniram & & \\
\hline & & Bagmoniram Siraja Khatun City Corporation Girl's High School & Shallow tube well \\
\hline 16 & Chawkbazar & Gul A Zar Begum City Corporation Girl's High School & Shallow tube well \\
\hline 17 & West Bakalia & West Bakalia Govt. Primary School & Deep tube well \\
\hline 18 & East Bakalia & East Bakalia City Corporation Girl's High School & Shallow tube well \\
\hline \multirow[t]{2}{*}{19} & South Bakalia & Char Chaktai City Corporation Boy’s High School & Shallow tube well \\
\hline & & Boluardhigi City Corporation Girl's High School & Shallow tube well \\
\hline \multirow[t]{3}{*}{20} & Dewan Bazar & & \\
\hline & & Lamabazar AAS City Corporation Boy’s High School & Shallow tube well \\
\hline & & Kadam Mubarak City Corporation Boy’s High School & Deep tube well \\
\hline \multirow[t]{2}{*}{21} & Jamal Khan & Jamal Khan Kusom Kumari City Corporation Girl's High School & Deep tube well \\
\hline & & Saleh Jahur City Corporation High School & Deep tube well \\
\hline \multirow[t]{2}{*}{22} & Enahet Bazar & & \\
\hline & & Postarpar City Corporation Boy’s High School & Shallow tube well \\
\hline \multirow[t]{2}{*}{23} & Uttar Pahartooly & Postarpar A Khatun City Corporation Girl’s High School & Deep tube well \\
\hline & & Patantooly Khan Shaheb City Corporation Girl's High School & Deep tube well \\
\hline 24 & North Agrabad & & \\
\hline 25 & Rampur & & \\
\hline 26 & North Halishahar & Halishahar Mohabbat Ali City Corporation High School & Deep tube well \\
\hline \multirow[t]{2}{*}{27} & South Agrabad & Al Nahian Primary School & Shallow tube well \\
\hline & & Patangooly City Corporation Boy's High School. & Deep tube well \\
\hline \multirow[t]{2}{*}{28} & Patantooly & & \\
\hline & & Patantooly City Corporation Girl's High School. & Deep tube well \\
\hline \multirow[t]{2}{*}{29} & West Madarbari & West Madarbari City Corporation Girl's High School. & Deep tube well \\
\hline & & East Madarbari City Corporation Girl’s High School. & Deep tube well \\
\hline \multirow[t]{2}{*}{30} & East Madarbari & & \\
\hline & & Primary Techer's Institute & Shallow tube well \\
\hline 31 & Alaran & Al-karan Nur Ahmed City Corporation Boy's High School. & Shallow tube well \\
\hline
\end{tabular}




\begin{tabular}{llll}
\hline Ward No. & Name of the ward & Well location & Remarks \\
\hline 32 & Anderkilla & Opornacharon City Corporation Girl's High School. & Deep tube well \\
& & Krishno Komari City Corporation Girl's High School. & Deep tube well \\
33 & Firingi Bazar & Al-karan Sultan Ahmed City Corporation Girl's High School. & Shallow tube well \\
34 & Patharghata & Patharghata Menaka City Corporation Boy's High School. & \\
35 & Boxir Hat & & Deep tube well \\
36 & Gosaildenga & Gosaildenga KBAN City Corporation Girl's High School. & \\
37 & North Middle Halishahar & & Deep tube well \\
38 & South Middle Halishahar & & Shallow tube well \\
39 & South Halishahar & Halishahar City Corporation Girl's High School. & Shallow tube well \\
\hline 40 & North Potenga & Potenga City Corporation Girl's High School. & South Potenga City Corporation High School. \\
41 & South Potenga & P
\end{tabular}

\subsection{Reduce Level of Ground Surface in Well Location by GPS}

$\mathrm{RL}$ of ground surface of well location at the reference of mean sea level by GPS has been shown in Table 3.

Table 3. Longitude, Latitude \& Elevation at the well location by GPS.

\begin{tabular}{ccccc}
\hline $\begin{array}{c}\text { Serial } \\
\text { No. }\end{array}$ & $\begin{array}{c}\text { War } \\
\text { d No. }\end{array}$ & Longitude & Latitude & $\begin{array}{c}\text { Elevation (RL of } \\
\text { ground surface w. } \\
\text { r. to MSL) }\end{array}$ \\
\hline 01 & 08 & $91^{0} 49^{\prime} 29.9^{\prime \prime}$ & $22^{0} 20^{\prime} 08.7^{\prime \prime}$ & $97^{\prime}$ \\
02 & 02 & $91^{0} 49^{\prime} 09.1^{\prime \prime}$ & $22^{0} 24^{\prime} 14.8^{\prime \prime}$ & $31^{\prime}$ \\
03 & 01 & $91^{0} 49^{\prime} 08.5^{\prime \prime}$ & $22^{0} 26^{\prime} 53.1^{\prime \prime}$ & $21^{\prime}$ \\
04 & 18 & $91^{0} 51^{\prime} 28.4^{\prime \prime}$ & $22^{0} 21^{\prime} 03.9^{\prime \prime}$ & $18^{\prime}$ \\
05 & 16 & $91^{0} 50^{\prime} 17.8^{\prime \prime}$ & $22^{0} 21^{\prime} 05.1^{\prime \prime}$ & $54^{\prime}$ \\
06 & 20 & $91^{0} 50^{\prime} 34.2^{\prime \prime}$ & $22^{0} 20^{\prime} 24.9^{\prime \prime}$ & $36^{\prime}$ \\
07 & 19 & $91^{0} 50^{\prime} 51.8^{\prime \prime}$ & $22^{0} 20^{\prime} 21.6^{\prime \prime}$ & $34^{\prime}$ \\
08 & 34 & $91^{0} 50^{\prime} 22.2^{\prime \prime}$ & $22^{0} 20^{\prime} 08.0^{\prime \prime}$ & $54^{\prime}$ \\
09 & 33 & $91^{0} 50^{\prime} 03.1^{\prime \prime}$ & $22^{0} 19^{\prime} 54.9^{\prime \prime}$ & $19^{\prime}$ \\
\hline
\end{tabular}

\begin{tabular}{ccccc}
\hline $\begin{array}{c}\text { Serial } \\
\text { No. }\end{array}$ & $\begin{array}{c}\text { War } \\
\text { d No. }\end{array}$ & Longitude & Latitude & $\begin{array}{c}\text { Elevation (RL of } \\
\text { ground surface w. } \\
\text { r. to MSL) }\end{array}$ \\
\hline 10 & 31 & $91^{0} 50^{\prime} 04.8^{\prime \prime}$ & $22^{0} 19^{\prime} 9.1^{\prime \prime}$ & $20^{\prime}$ \\
11 & 30 & $91^{0} 49^{\prime} 37.3^{\prime \prime}$ & $22^{0} 19^{\prime} 56.3^{\prime \prime}$ & $18^{\prime}$ \\
12 & 23 & $91^{\circ} 49^{\prime} 02.3^{\prime \prime}$ & $22^{0} 20^{\prime} 17.2^{\prime \prime}$ & $36^{\prime}$ \\
13 & 41 & $91^{0} 47^{\prime} 36.7^{\prime \prime}$ & $22^{0} 15^{\prime} 24.5^{\prime \prime}$ & $44^{\prime}$ \\
14 & 40 & $91^{0} 48^{\prime} 28.3^{\prime \prime}$ & $22^{0} 14^{\prime} 16.7^{\prime \prime}$ & $48^{\prime}$ \\
15 & 13 & $91^{0} 48^{\prime} 31.8^{\prime \prime}$ & $22^{0} 21^{\prime} 41.6^{\prime \prime}$ & $76^{\prime}$ \\
16 & 27 & $91^{\circ} 48^{\prime} 07.2^{\prime \prime}$ & $22^{0} 19^{\prime} 25.9^{\prime \prime}$ & $27^{\prime}$ \\
17 & 15 & $91^{0} 49^{\prime} 21.6^{\prime \prime}$ & $22^{0} 21^{\prime} 07.6^{\prime \prime}$ & $61^{\prime}$ \\
18 & 10 & $91^{\circ} 48^{\prime} 30.2^{\prime \prime}$ & $22^{0} 20^{\prime} 18.5^{\prime \prime}$ & $40^{\prime}$ \\
\hline
\end{tabular}

\subsection{Depth of Water Table in Three Different Seasons from Mean Sea Level}

Depth of water table measured in three different seasons from ground level \& adjustment of depth from mean sea level has been shown in Table 4 .

Table 4. Depth of water table in three different seasons from mean sea level.

\begin{tabular}{|c|c|c|c|c|c|}
\hline Ward No & Name of Ward & Well location & $\begin{array}{l}\text { At the beginning } \\
\text { of winter season }\end{array}$ & At drought season & $\begin{array}{l}\text { At the beginning of } \\
\text { rainy season }\end{array}$ \\
\hline 01 & South Pahartali & $\begin{array}{l}\text { Fatehabad Bohumuki City Corporation Girl's } \\
\text { High School. }\end{array}$ & $\left(21^{\prime}-32^{\prime}\right)=-11^{\prime}$ & $\left(21^{\prime}-39^{\prime}\right)=-18^{\prime}$ & $\left(21^{\prime}-34^{\prime}\right)=-13^{\prime}$ \\
\hline 02 & Jalabad & Kulgaon City Corporation High School. & $\left(31^{\prime}-36^{\prime}\right)=-5^{\prime}$ & $\left(31^{\prime}-42^{\prime}\right)=-11^{\prime}$ & $\left(31^{\prime}-39^{\prime}\right)=-8^{\prime}$ \\
\hline 08 & Sulakbahar & $\begin{array}{l}\text { Hossain Ahmed Chowdhury City Corporation } \\
\text { School. }\end{array}$ & $\left(97^{\prime}-78^{\prime}\right)=19^{\prime}$ & $\left(97^{\prime}-82^{\prime} 4^{\prime \prime}\right)=14^{\prime} 8^{\prime \prime}$ & $\left(97^{\prime}-75^{\prime} 10^{\prime \prime}\right)=21^{\prime \prime \prime}$ \\
\hline 10 & North Kattali & Kattali City Corporation Girl's High School. & $\left(40^{\prime}-42^{\prime} 6^{\prime \prime}\right)=-2^{\prime} 6^{\prime \prime}$ & $\left(40^{\prime}-45^{\prime}\right)=-5^{\prime}$ & $\left(40^{\prime}-41^{\prime}\right)=-1^{\prime}$ \\
\hline 13 & Pahartali & New Jawtala Primary School & $\left(76^{\prime}-48^{\prime}\right)=28^{\prime}$ & $\left(76^{\prime}-52^{\prime}\right)=24^{\prime}$ & $\left(76^{\prime}-48^{\prime} 6^{\prime \prime}\right)=27^{\prime} 6^{\prime \prime}$ \\
\hline 15 & Bagmoniram & $\begin{array}{l}\text { Bagmoniram Abdur Rashid City Corporation } \\
\text { Boy's High School. }\end{array}$ & $\left(61^{\prime}-113^{\prime}\right)=-52^{\prime}$ & $\left(61^{\prime}-120^{\prime}\right)=-59^{\prime}$ & $\left(61^{\prime}-108^{\prime}\right)=-47^{\prime}$ \\
\hline 16 & Chawk Bazar & $\begin{array}{l}\text { Gul A Zar Begum City Corporation Girl's High } \\
\text { School. }\end{array}$ & $\left(54^{\prime}-35^{\prime}\right)=19^{\prime}$ & $\left(54^{\prime}-41^{\prime}\right)=13^{\prime}$ & $\left(54^{\prime}-38^{\prime}\right)=16^{\prime}$ \\
\hline 18 & East Bakalia & $\begin{array}{l}\text { East Bakalia City Corporation Girl’s High } \\
\text { School. }\end{array}$ & $\left(18^{\prime}-37^{\prime} 6^{\prime \prime}\right)=-19^{\prime} 6^{\prime \prime}$ & $\left(18^{\prime}-41^{\prime}\right)=-23^{\prime}$ & $\left(18^{\prime}-31^{\prime}\right)=-13^{\prime}$ \\
\hline 19 & South Bakalia & $\begin{array}{l}\text { Char Chaktai City Corporation Boy’s High } \\
\text { School. }\end{array}$ & $\left(34^{\prime}-29^{\prime} 7^{\prime \prime}\right)=4^{\prime} 5^{\prime \prime}$ & $\left(34^{\prime}-24^{\prime} 6^{\prime \prime}\right)=9^{\prime} 6^{\prime \prime}$ & $\left(34^{\prime}-22^{\prime} 6^{\prime \prime}\right)=11^{\prime} 6^{\prime \prime}$ \\
\hline 20 & Dewan Bazar & $\begin{array}{l}\text { Lama Bazar AA S City Corporation Boy's High } \\
\text { School. }\end{array}$ & $\left(36^{\prime}-33^{\prime}\right)=3^{\prime}$ & $\left(36^{\prime}-41^{\prime} 6^{\prime \prime}\right)=-5^{\prime} 6^{\prime \prime}$ & $\left(36^{\prime}-33^{\prime} 6^{\prime \prime}\right)=2^{\prime} 6^{\prime \prime}$ \\
\hline 23 & Uttar Pahartooly & Posterpar City Corporation Boy's High School. & $\left(36^{\prime}-70^{\prime}\right)=-34^{\prime}$ & $\left(36^{\prime}-75^{\prime} 6^{\prime \prime}\right)=-39^{\prime} 6^{\prime \prime}$ & $\left(36^{\prime}-71^{\prime}\right)=-35^{\prime}$ \\
\hline 27 & South Agrabad & Al Nahian Primary School. & $\left(27^{\prime}-23^{\prime}\right)=4^{\prime}$ & $\left(27^{\prime}-27^{\prime}\right)=0^{\prime}$ & $\left(27^{\prime}-25^{\prime} 6^{\prime \prime}\right)=1^{\prime} 6^{\prime \prime}$ \\
\hline 30 & East Madarbari & Primary Teacher’s Institute. & $\left(18^{\prime}-12^{\prime} 6^{\prime \prime}\right)=5^{\prime} 6^{\prime \prime}$ & $\left(18^{\prime}-18^{\prime}\right)=0^{\prime}$ & $\left(18^{\prime}-15^{\prime}\right)=3^{\prime}$ \\
\hline 31 & Alkaran & $\begin{array}{l}\text { Alkaran Nur Ahmed City Corporation Boy's } \\
\text { High School. }\end{array}$ & $\left(20^{\prime}-18^{\prime} 5^{\prime \prime}\right)=17^{\prime \prime}$ & $\left(20^{\prime}-25^{\prime} 9^{\prime \prime}\right)=-5^{\prime} 9^{\prime \prime}$ & $\left(20^{\prime}-20^{\prime}\right)=0^{\prime}$ \\
\hline 33 & Firingi Bazar & $\begin{array}{l}\text { Alkaran Sultan Ahmed City Corporation Girl's } \\
\text { High School. }\end{array}$ & $\left(19^{\prime}-17^{\prime}\right)=2^{\prime}$ & $\left(19^{\prime}-23^{\prime}\right)=-4^{\prime}$ & $\left(19^{\prime}-20^{\prime}\right)=-1^{\prime}$ \\
\hline 34 & Patharghata & $\begin{array}{l}\text { Patharghata Menaka City Corporation Boy's } \\
\text { High School. }\end{array}$ & $\left(54^{\prime}-45^{\prime}\right)=9^{\prime}$ & $\left(54^{\prime}-52^{\prime} 7 "\right)=1{ }^{\prime \prime}$ & $\left(54^{\prime}-49^{\prime} 6^{\prime \prime}\right)=4^{\prime} 6^{\prime \prime}$ \\
\hline 40 & North Poenta & Potenga City Corporation Girl's High School. & $\left(48^{\prime}-30^{\prime}\right)=18^{\prime}$ & $\left(48^{\prime}-30^{\prime}\right)=18^{\prime}$ & $\left(48^{\prime}-22^{\prime}\right)=26^{\prime}$ \\
\hline 41 & South Poenta & South Potenga City Corporation High School. & $\left(44^{\prime}-25^{\prime}\right)=19^{\prime}$ & $\left(44^{\prime}-25^{\prime} 5 "\right)=18^{\prime} 7^{\prime \prime}$ & $\left(44^{\prime}-21^{\prime}\right)=23^{\prime}$ \\
\hline
\end{tabular}




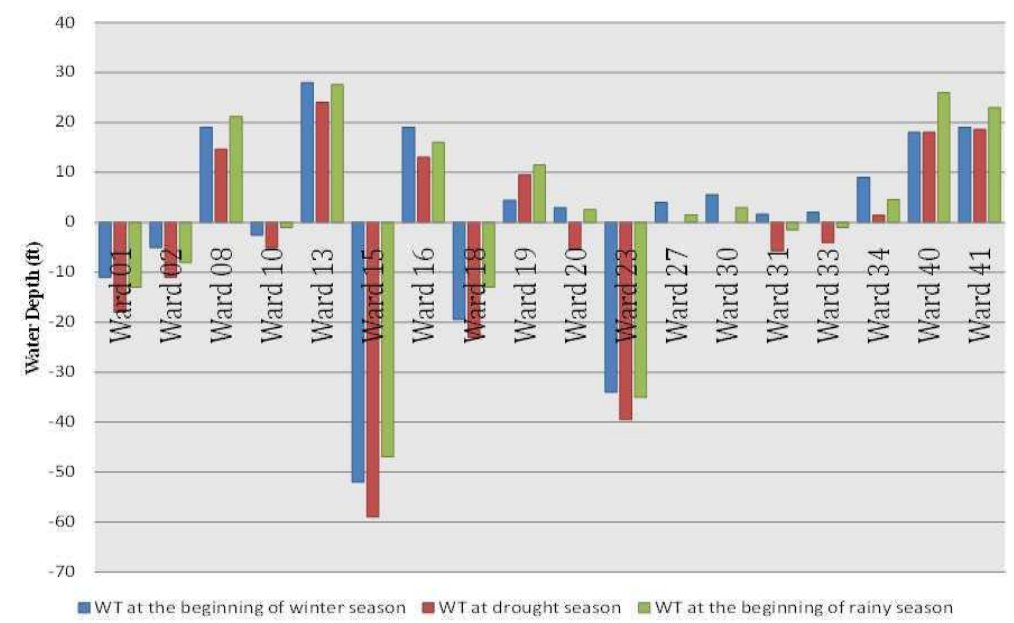

Figure 6. Graphical representation of seasonal WT variation for various wards.

\section{Water Quality of Investigated Well}

\subsection{Test Result of Water Quality Parameter}

Laboratory test has been conducted on water collected from well location. The comparison of test result with standard values has been shown in Table 5 .

Table 5. Test results for different parameters.

\begin{tabular}{|c|c|c|c|c|c|c|}
\hline Ward No & pH & $\mathrm{CO}_{2}(\mathrm{mg} / \mathrm{l})$ & Iron (mg/l) & Alkalinity (mg/l) & $\begin{array}{l}\text { Arsenic } \\
(\mathrm{mg} / \mathrm{l})\end{array}$ & Chloride (mg/l) \\
\hline WHO & $6.5-8.5$ & --- & $0.1-1.0$ & --- & 0.01 & 250 \\
\hline Bangladesh & $6.5-8.5$ & --- & $0.3-1.0$ & $200-500$ & 0.05 & $200-300$ \\
\hline 01 & 6.97 & 37.5 & 0.1 & 110 & Nil & 7 \\
\hline 02 & 6.98 & 32.5 & 0.1 & 120 & Nil & 10 \\
\hline 08 & 6.88 & 15 & 0.001 & 30 & Nil & 20 \\
\hline 10 & 6.99 & 22.5 & 0.1 & 80 & Nil & 15 \\
\hline 13 & 7.10 & 20 & 0.001 & 70 & Nil & 5 \\
\hline 15 & 7.20 & 37.5 & 0.1 & 110 & Nil & 35 \\
\hline 16 & 6.86 & 25 & 0.001 & 50 & Nil & 15 \\
\hline 18 & 7.02 & 30 & 0.2 & 130 & Nil & 62 \\
\hline 19 & 7.09 & 35 & 0.2 & 130 & Nil & 85 \\
\hline 20 & 7.19 & 62.5 & 0.1 & 80 & Nil & 40 \\
\hline 23 & 7.32 & 25 & 0.001 & 320 & Nil & 950 \\
\hline 27 & 7.45 & 22.5 & 0.3 & 350 & $<0.01$ & 1000 \\
\hline 30 & 6.95 & 20 & 0.001 & 120 & Nil & 30 \\
\hline 31 & 7.85 & 72.5 & 0.2 & 290 & Nil & 145 \\
\hline 33 & 7.11 & 55 & 0.1 & 230 & Nil & 143 \\
\hline 34 & 7.08 & 30 & 0.4 & 280 & Nil & 70 \\
\hline 40 & 7.38 & 95 & 0.8 & 420 & $<0.01$ & 750 \\
\hline 41 & 7.21 & 55 & 0.001 & 270 & Nil & 20 \\
\hline
\end{tabular}

\section{Conclusion and Discussions}

Ground water table in Chittagong City Corporation area is in a position where day by day the water table is lowering. In this project, out aim was to find out the seasonal variation of the ground water table. For this purpose we have to select the shallow well instead of deep well by considering the limitation we have. Thus within 41 wards in Chittagong City Corporation area, the location selected is City Corporation School, where in only 18 wards shallow well was found. By considering this limitation we investigate the ground water table in those 18 wards where there is presence of shallow tube well.

Conclusion: The concluding remarks that can be derived from the investigation have been presented below-

a. Water tables are different in different wards. But nearly similar in adjacent wards.

b. During field investigation, it was found that within 50 well locations in 41 wards; only 21 shallow tube well is present. As majority of the location can't pump water through shallow tube well.

c. Majority of the shallow tube well that can pump water has a depth of (50'-110'). 
d. The overall view shows that, in almost every ward, to find fresh water deep tube well is must as shallow tube well can't frequently pump water.

e. Through the water quality test in the laboratory, it has been found that, majority of shallow tube well water tested having $\mathrm{pH}, \mathrm{CO}_{2}$, Iron, Alkalinity, Chloride concentration \& Arsenic within the limit suggested by WHO and Bangladesh standard.

f. From the investigation it is clear that, ground water table is lowering day by day and it is lower most at the drought season. At the beginning of rainy season when it started to rain the water table comes up.

g. Ground water through shallow tube well is not sufficient to fulfill the required demand for the general people as it is becoming out of reach through shallow well day by day.

h. Chittagong is the $2^{\text {nd }}$ most important city of Bangladesh. But still the depth of water table has not been measured by any organization.

i. Depth of water table with respect to mean sea level is quite lower in ward no-15 \& 23.

j. Depth of water table with respect to mean sea level is quite higher in ward no-08, 13, 40, \& 41.

\section{References}

[1] M. A. Aziz, Water Supply Engineering, Hafiz Book Centre, First Edition, 1975.
[2] M. Feroze Ahmed and Md. Mujibur Rahman, Water Supply \& Sanitation, Rural and low income urban communities, Centre for Water Supply \& Water management BUET, Dhaka, Bangladesh, a Dania funded project, ITNBANGLADESH, First Edition, 2000

[3] H. M. Raghunath, Ground Water, New Age International (P) Limited, Publishers, New Delhi, 1996.

[4] Banglapedia.search.com.bd/Map.

[5] WHO Guidelines for drinking water quality. $2^{\text {nd }}$ edition. Volume 2, 1996.

[6] S. K. Garg. Water Supply Engineering, Khanna Publishers, 2-B Nath Market, Nai Sarak, Delhi-1100.

[7] Pamela J.W. Gore, Georia Perimeter College, Groundwater, 1996.

[8] http://E:IGWT \grndh2o.htm . (24 ${ }^{\text {th }}$ October 2010)

[9] http://E:IGWTVhydro.html . $\left(24^{\text {th }}\right.$ October 2010)

[10] http://ga.water.usgs.gov/edu/gwdepletion.html.(28 $8^{\text {th }}$ October 2010)

[11] "Learn More: Groundwater", Colombia Water Center.

[12] Parkison, Spilker, the Global Positioning System, 2006.

[13] Nathaniel, Bowditch. The American Practical Navigator. Chapter II Satellite Navigation.

[14] List of school from Chittagong City Corporation.

[15] Location \& depth of deep tube well of CWASA from CWASA (2011). 